\title{
CYT387, a potent IKBKE inhibitor, suppresses human glioblastoma progression by activating the Hippo pathway
}

Xin Wang ${ }^{1 *+}$, Jie $\mathrm{Lu}^{2+}{ }^{\circ}$, Jing $\mathrm{Li}^{3}$, Yang Liu ${ }^{4}$, Gaochao Guo ${ }^{4}$ and Qiang Huang ${ }^{5,6^{*}}$

\begin{abstract}
Recent studies have showed that IKBKE is overexpressed in several kinds of cancers and that IKBKE-knockdown inhibits tumor progression. In this article, we first verified that two glioblastoma cell lines, U87-MG and LN-229, were sensitive to CYT387 by measuring the half maximal inhibitory concentration (IC50) with a CCK-8 assay and then demonstrated that CYT387, as a potent IKBKE inhibitor, suppressed glioblastoma cell proliferation, migration and invasion. Additionally, CYT387 induced cell apoptosis and arrested the cell cycle at the G2/M checkpoint in vitro. Furthermore, we showed that CYT387 did not simply inhibit IKBKE activity but also decreased IKBKE expression at the protein level rather than at the mRNA level. We discovered that CYT387 restrained malignant tumor progression by activating the Hippo pathway in vitro. By coimmunoprecipitation (Co-IP), we showed that IKBKE interacted with TEAD2 and YAP1, thus accelerating TEAD2 and YAP1 transport into the nucleus. In subsequent in vivo experiments, we found that CYT387 inhibited subcutaneous nude mouse tumor growth but had little impact on intracranial orthotopic xenografts, probably due to a limited ability to penetrate the blood-brain barrier (BBB). These results suggest that CYT387 has potential as a new antiglioblastoma drug, but an approach to allow passage through the blood-brain barrier (BBB) is needed.
\end{abstract}

Keywords: CYT387, IKBKE, Glioblastoma, Hippo pathway

\section{Introduction}

Glioblastoma, a common intracranial primary malignant glioma, has high recurrence, morbidity and mortality rates. Although the most effective therapy includes surgical resection, radiotherapy with concomitant and adjuvant temozolomide has been widely adopted, however, the poor patient prognosis, which is represented by the 5 -year survival rate of $9.8 \%$ and median survival time of

\footnotetext{
*Correspondence: wangoncologist@126.com; huangqiang@tmu.edu.cn

tXin Wang and Jie Lu contributed equally to this work

${ }^{1}$ Department of Radiation Oncology, Shandong Cancer Hospital

and Institute, Shandong First Medical University and Shandong Academy of Medical Sciences, Jinan, Shandong, China

${ }^{5}$ Department of Neurosurgery, Tianjin Medical University General Hospital, Tianjin, China

Full list of author information is available at the end of the article
}

14.6 months, remains to be improved [1,2]. Due to the high heterogeneity, low immunogenicity and immunosuppressive microenvironment, the effective immune checkpoint inhibitor therapy and tumor target therapy for many tumors have little benefit in glioblastoma [3, 4]. Glioblastoma has become an intractable disease in neurosurgery, emphasizing that there is an urgent need to delineate the underlying molecular mechanisms and identify new treatment strategies.

IKBKE, also called IKKe or IKKi, belongs to the IKB kinase (IKK) family that induces noncanonical NF- $\mathrm{KB}$ signaling $[5,6]$. In prior studies, IKBKE has been demonstrated to be a novel oncogene in breast cancer and was shown to be amplified in over $30 \%$ of breast cancer cases [7]. Recent studies have also shown that IKBKE is overexpressed in glioma [8-10], ovarian cancer [10], prostate 
cancer $[11,12]$, non-small cell lung cancer $[13,14]$, gastric cancer [15] and renal clear cell carcinoma [16]. These studies have revealed that IKBKE has close relationships with cancer pathological grade or clinical stage in glioma [9], ovarian cancer [10], and lung squamous cell cancer $[14,17]$ and induces tumor chemoresistance in ovarian cancer [11] and non-small cell lung cancer [13]. In addition, overexpression of IKBKE results in malignant cell transformation [7, 14]. Li et al. [9] showed that silencing IKBKE inhibited glioma proliferation in vitro and in vivo, indicating that IKBKE can contribute to glioma progression. Taken together, these data indicate the importance of IKBKE in tumorigenesis and suggest that inhibiting IKBKE expression may represent a new approach for the treatment of malignancy [18].

CYT387 (also named momelotinib), traditionally considered a JAK1 and JAK2 inhibitor, has been used in the clinical treatment of myelofibrosis. However, an increasing number of extensive studies have focused on its usage in myeloproliferative neoplasms [19] and other tumors. Lue et al. [20] reported that the combined use of CYT387 (a JAK/STAT inhibitor) and dasatinib (an Src inhibitor) synergistically reduced cell proliferation and increased apoptosis in renal cell carcinoma. Hu et al. [21] pointed out that CYT387 in combination with cetuximab (an EGFR inhibitor) effectively inhibited non-small cell lung cancer proliferation, especially that of EGFR inhibitorresistant tumors. Zhu et al. [22] revealed that CYT387 could suppress IKBKE kinase activity in an in vitro kinase assay, thus blocking KRAS-dependent lung cancer cell growth. Barbie et al. [23] showed that CYT387, as a TBK1/IKBKE/JAK inhibitor, inhibited triple-negative breast cancer proliferation by suppressing the NF- $\mathrm{KB}$ and STAT3 activation induced by IKBKE; however, there was almost no inhibitory effect on cancer growth if only JAK activity was suppressed. Generally, CYT387 can inhibit both IKBKE activation and JAK/STAT activation to regulate tumor growth via a complicated pathway. However, the specific function of CYT387 in glioblastoma remains to be elucidated.

The Hippo pathway regulates cell proliferation, differentiation, tissue development and stemness in conjunction with inputs from the intracellular and extracellular microenvironments, including cell contact, cell polarity, and mechanotransduction [24, 25]. Dysregulation of the Hippo pathway can cause cancer development. In recent studies, Ji et al. [26] pointed out that the LATS1 expression level was remarkably decreased in glioma tissues and had a close relationship with the tumor grade and prognosis of patients. Orr et al. [27] showed that YAP1 was overexpressed in high-grade glioma and that knocking down YAP1 expression obviously inhibited glioma cell line proliferation, suggesting that YAP1 plays an important role in glioma progression. These data indicate that inactivation of the Hippo pathway leads to glioblastoma progression and that the Hippo pathway is a candidate for therapeutic manipulation.

In this article, we first demonstrated that CYT387, as an IKBKE inhibitor, inhibited glioma cell proliferation, migration, and invasion in vitro; accelerated cell apoptosis; and arrested the cell cycle at the G2/M checkpoint. In addition, we verified that CYT387 increased Hippo pathway activity to inhibit glioma malignancy and that IKBKE directly interacted with YAP1 and TEAD2, as determined by using coimmunoprecipitation (co-IP). Additionally, inhibition of IKBKE suppressed YAP1 and TEAD2 translocation into the nucleus. Moreover, we showed that CYT387 could suppress glioblastoma growth in a subcutaneous nude mouse model but had little impact on intracranial orthotopically implanted tumors. Our data indicate that CYT387 may become a new anticancer drug of interest for glioblastoma treatment, but its limited ability to penetrate the blood-brain barrier (BBB) needs to be addressed.

\section{Materials and methods \\ Cell culture, transfection and antibodies}

The human glioblastoma cell lines U251 and LN229 came from the Institute of Biochemistry and Cell Biology (Shanghai, China). HEK293 cells were from the Institute of Biochemistry and Cell Biology (Shanghai, China). All cells were maintained in Dulbecco's modified Eagle's medium (DMEM, Gibco, USA) supplemented with 10\% fetal bovine serum (Gibco, USA), and cultured at $37^{\circ} \mathrm{C}$ in $5 \% \mathrm{CO}_{2}$. We established an IKBKE-shRNA lentiviral vector from GeneChem (Shanghai, China) with the sequence of 5'-GCATCATCGAACGGCTAAATA-3'. A GFP scrambled lentiviral vector with the sequence of $5^{\prime}-\mathrm{TTC}$ TCCGAACGTGTCACGTTTC-3' was used as the negative control. The shRNAs were transfected according to the manufacturer's instructions. The Flag-IKBKE plasmid was purchased from Addgene (USA). The HA-TEAD2 and HA-YAP1 plasmids were from Hanbio Biotechnology (Shanghai, China). The IKBKE-overexpress lentivirus and its empty vector virus were from GeneChem (Shanghai, China). We selected Lipofectamine 3000 (thermo fisher scientific, USA) as transfection medium and the process of transfection was according to the manufacturer's instructions. IKBKE rabbit mAb (No.2905,WB 1:1000; IP 1:100), c-myc rabbit mAb (No.13987,WB 1:1000), MMP9 rabbit $\mathrm{mAb}$ (No.13667,WB 1:1000), YAP1 mouse mAb (No.12395,WB 1:1000; IHC 1:400; IP 1:100), Bcl-2 rabbit mAb (No.2870,WB 1:1000), Phospho-YAP (Ser127) rabbit mAb (No.13008,WB 1:1000; IHC 1:2000), HA-Tag rabbit mAb (No.3724,WB 1:1000; IP 1:50), DYKDDDDKTag (Flag) rabbit mAb (No.14793,WB 1:1000; IP 1:50) and 
Axl rabbit mAb (No.8661,WB 1:1000;IHC 1:300) were purchased from Cell Signaling Technology (USA). IKBKE rabbit polyclonal antibody (ab7891,IHC 1:100), Cdk1 rabbit mAb (ab133327,WB 1:10000;IHC 1:300), Cdc25c rabbit $m A b$ (ab32444,WB 1:2000), caspase-9 rabbit $m A b$ (ab202068,WB 1:2000), Bax rabbit mAb (ab32503,WB 1:2000), CyclinB $B_{1}$ rabbit mAb (ab32053,WB 1:5000;IHC 1:100), Cyclin $\mathrm{A}_{2}$ rabbit mAb (ab181591,WB 1:2000), c-myc rabbit mAb (ab32072,IHC 1:500), CyclinD $D_{1}$ rabbit mAb (ab134175,WB:1:10000), TEAD2 rabbit polyclonal antibody (ab83670, WB 1:500) were purchased from Abcam (USA). TEAD2 rabbit polyclonal antibody (sc-67115, IP 1:50) was from Santa Cruz (USA). LATS2 rabbit polyclonal antibody (20276-1-AP, WB 1:500; IHC 1:50) were purchased from proteintech (USA). GAPDH mouse mAb (WB 1:2000) was from ZSGB-Bio (Beijing, China).

\section{Protein extraction and western blot analysis}

The cell total protein was extracted after treatment of CYT387, plasmids or IKBKE-shRNA using RIPA lysis buffer with protease and phosphatase inhibitor (MCE USA). The homogenates were clarified by centrifugation at $4{ }^{\circ} \mathrm{C}$ for $15 \mathrm{~min}$ at $12,000 \mathrm{rpm}$ after cleavage by RIPA for $15 \mathrm{~min}$, and the protein concentration was measured by BCA assay kit (Beyotime, Shanghai, China). At least $20 \mu \mathrm{g}$ protein mixed with $4 \times$ loading buffer was added into spacer gel and then separated by sodium dodecylsulphate-polyacrylamide gel electrophoresis (SDS-PAGE). The protein bands were electrotransferred to PVDF membranes (Millipore, USA). Primary antibodies were incubated at $4{ }^{\circ} \mathrm{C}$ for overnight then HRP-conjugated secondary antibody (1:3000 dilution, ZSGB-Bio, Beijing, China) was used for $1 \mathrm{~h}$ at room temperature. The bands were detected by the G:BOX (Syngene Company, UK) using Chemiluminescent HRP Substrate (Millipore USA).

\section{RNA extraction and real-time RT-PCR analysis}

Total RNA of glioblastoma cells (U87-MG and LN-229) after treatment with CYT387 in dose- and time-dependent manners was extracted by TRIzol reagent (Invitrogen, USA) following the manufacturer's protocols and then reverse transcription was performed using GoScript ${ }^{\mathrm{TM}}$ Reverse Transcription System (Promega, USA). The quantitative real-time PCR was finished by GoTaq qPCR Master Mix (Promega, USA) according to the supplier's instructions. The reaction conditions were as follows: $95^{\circ} \mathrm{C}$ for 5 min and 40 cycles of $95^{\circ} \mathrm{C}$ for $12 \mathrm{~s}$ and $60^{\circ} \mathrm{C}$ for $40 \mathrm{~s}$. The primers were synthesized by GENEWIZ (USA). The sequences of the primers were as follows: GAPDH: 5'-GGAGCGAGATCCCTCCAAAAT-3' (Forward primer) and 5'-GGCTGTTGTCATACTTCT
CATGG-3' (Reverse primer); IKBKE: 5'-GAGAAGTTC GTCTCGGTCTATGG-3' (Forward primer) and 5'-TGC ATGGTACAAGGTCACTCC-3' (Reverse primer); TEAD2: 5'-GCCTCCGAGAGCTATATGATCG-3' (Forward primer) and 5'-TCACTCCGTAGAAGCCACCA-3' (Reverse primer); YAP1: 5'-TAGCCCTGCGTAGCC AGTTA-3' (Forward primer) and 5'-TCATGCTTAGTC CACTGTCTGT-3' (Reverse primer). GAPDH was used as internal control.

\section{Clone formation assay}

U87-MG and LN-229 cells were seed in six-well plates $\left(2 \times 10^{3} /\right.$ well $)$ divided into three groups as blank control, negative control (DMSO) and drug group (CYT387 with concentration of $6 \mu \mathrm{M})$. Growth medium was changed every 6 days. After 12 days, cells were fixed in 4\% paraformaldehyde for $15 \mathrm{~min}$ and stained with crystal violet for $30 \mathrm{~min}$. Colonies were scored after photographed.

\section{CCK-8 assay}

For $\mathrm{IC}_{50}$ measurement, we seeded U87-MG and LN-229 cells $(5000 / 50 \mathrm{ul} /$ well $)$ into 96 -well plates on the first day. After cells were adherent, we again added 50ul/ well medium with different concentration of CYT387 to achieve the final drug concentration gradient of $0.5 \mu \mathrm{M}$, $1 \mu \mathrm{M}, 2 \mu \mathrm{M}, 4 \mu \mathrm{M}, 8 \mu \mathrm{M}, 16 \mu \mathrm{M}, 32 \mu \mathrm{M}$ and $64 \mu \mathrm{M}$. After treatment with CYT387 for 24, 48, and $72 \mathrm{~h}, 10 \mu \mathrm{l}$ CCK8 reagent (dojindo, Japan) was mixed into each well and then 96-well plate was incubated for $2 \mathrm{~h}$ at $37^{\circ} \mathrm{C}$. The O.D. value was measured by Microplate reader at the wavelength of $450 \mathrm{~nm}$.

For proliferative curve measurement, glioblastoma cells with normal medium, DMSO medium and $6 \mu \mathrm{M}$ CYT387 medium were seeded $(2000 / 100 \mu \mathrm{l} /$ well $)$ into 96-well plates. From first day to fifth day, $10 \mu \mathrm{l} \mathrm{CCK-8}$ reagent (dojindo, Japan) was added into each well. The O.D. value was measured after incubated for $2 \mathrm{~h}$ at $37^{\circ} \mathrm{C}$ in a $5 \% \mathrm{CO}_{2}$ atmosphere.

\section{Wound healing assays}

U87-MG and LN-229 cells were seeded in six-well plate and a straight wound was created with a sterile $100 \mu \mathrm{l}$ pipette tip. Then we respectively added DMSO and CYT387 in DMSO group and drug group, making the drug concentration of $6 \mu \mathrm{M}$. After treatment for $24 \mathrm{~h}$ and $48 \mathrm{~h}$, the wound healing area was detected by an inverted microscope.

\section{Transwell assay}

Before experiment begun, matrigel with 3 times volume serum-free DMEM (total $80 \mu \mathrm{l} /$ well) was coated on the upper surface of chamber. Then place it at $37^{\circ} \mathrm{C}$ for $30 \mathrm{~min}$, waiting for matrigel solidification. Then the 
cells $\left(5 \times 10^{4} /\right.$ well $)$ were seeded into the transwell chambers with $200 \mu \mathrm{l}$ serum-free DMEM while outer space was filled with $500 \mu \mathrm{l}$ serum DMEM. After incubated at $37{ }^{\circ} \mathrm{C}$ in a $5 \% \mathrm{CO}_{2}$ atmosphere for $48 \mathrm{~h}$, cells across the chamber membrane was fixed with $4 \%$ paraformaldehyde for $15 \mathrm{~min}$, then stained with crystal violet for $5 \mathrm{~min}$, counted and imaged under the microscope.

\section{Cell apoptosis assays and cell cycle analysis}

Before cell apoptosis assay, the cells were treated with CYT387 with concentration of $6 \mu \mathrm{M}$ for $24 \mathrm{~h}$. Cells were trypsinized without EDTA, washed with PBS twice and then stained using the Annexin V-FITC Apoptosis Detection kit from KeyGen Biotech (Nanjing, China) according to the manufacturer's instructions. Flow cytometry analysis was finished by a FACS flow cytometer (BectonDickinson). Data were analyzed by CellQuest software. Before cell cycle analysis, the U87-MG and LN-229 cells was treated with CYT387 at the concentration of $6 \mu \mathrm{M}$ for $72 \mathrm{~h}$. Then the following protocols were performed as the previous article [9].

\section{Co-immunoprecipitation (co-IP)}

Co- immunoprecipitation (co-IP) was carried out as described previously [28].

\section{Animal studies}

Before animal studies, CYT387 purchased from selleck (USA) was dissolved in NMP (1-methyl-2-pyrrolidinone) to finally get the concentration of $120 \mathrm{mg} / \mathrm{ml}$. Next, the CYT387 was diluted with 0.14 M Captisol (MCE, USA) to a concentration of $6 \mathrm{mg} / \mathrm{ml}$. The tumor subcutaneous experiments method was carried out as described previously [9]. After subcutaneous tumour was shaped, the nude mice were fed with CYT387 (100 mg/kg/day).

For intracranial orthotopic model, U87-MG transfected with luciferase-expressing lentivirus was injected intracranially into 6-week-old BALB/c-nu mice. After 7 days, mice started to be fed with CYT387 $(100 \mathrm{mg} / \mathrm{kg} /$ day). The weight of mice was monitored every 2 days and the luminescence imaging of intracranial tumour was measured using an IVIS Lumina Imaging System (Xenogen) every 7 days.

\section{Immunohistochemical staining}

After mice were sacrificed for subcutaneous and intracranial tumour, we made specimens embedded with paraffin. Then the paraffin-embedded tumours were sectioned and dewaxed. After antigen retrieval using $10 \mathrm{mmol} / \mathrm{l}$ citrate buffer, sections were incubated with $3 \% \mathrm{H}_{2} \mathrm{O}_{2}$ and blocked with 5\% BSA. Then the sections were added with primary antibodies at $4{ }^{\circ} \mathrm{C}$ overnight. After rewarming at room temperature the next day, the sections were incubated with secondary antibodies using two-step polymer HRP detection system (ZSGBBIO, Beijing, China). The samples were colourated with DBA Kit (ZSGB-BIO, Beijing, China) and then counterstained with haematoxylin. After dehydration and sealing piece with neutral gum, the samples were detected and photographed by microscope (Olympus Japan).

\section{Statistical analysis}

All data were repeated at least three times. Quantitative data are shown as the mean \pm standard deviation (SD). We used SPSS software (version 16.0) for the statistical analyses and $\mathrm{P}<0.05$ was considered statistically significant.

\section{Results}

\section{CYT387 remarkably inhibits glioblastoma cell proliferation} in vitro

To evaluate the sensitivity of glioblastoma cells to CYT387 in vitro, two glioblastoma cell lines that highly express IKBKE, U87-MG and LN-229 [10], were treated with a concentration gradient of CYT387 $(0.5 \mu \mathrm{M}$ to $64 \mu \mathrm{M})$ to measure the half maximal inhibitory concentration (IC50) with a CCK-8 assay. A doseresponse curve with data points for $24 \mathrm{~h}, 48 \mathrm{~h}$, and $72 \mathrm{~h}$ of drug treatment is shown in Fig. 1a. The IC50 values of U87-MG cells were $6.395 \pm 1.127 \mu \mathrm{M}$ for $72 \mathrm{~h}$, $17.68 \pm 2.94 \mu \mathrm{M}$ for $48 \mathrm{~h}$, and $24.87 \pm 3.63 \mu \mathrm{M}$ for $24 \mathrm{~h}$, and those of LN229 cells were $5.139 \pm 0.501 \mu \mathrm{M}$ for $72 \mathrm{~h}, 17.18 \pm 2.61 \mu \mathrm{M}$ for $48 \mathrm{~h}$, and $25.46 \pm 3.59 \mu \mathrm{M}$ for $24 \mathrm{~h}$. These data demonstrated that glioblastoma cells were sensitive to CYT387, especially after $72 \mathrm{~h}$ of treatment. Then, we carried out a CCK- 8 assay to investigate the effect of CYT387 on glioblastoma cell proliferation using a drug concentration of $5 \mu \mathrm{M}$ and incubating the cells for 5 days. The results (Fig. 1b) showed that the viability of U87-MG and LN229 cells treated with CYT387 was dramatically decreased compared with that of the cells in the blank control and DMSO (drug solvent) groups. Additionally, a colony formation assay was used to test whether CYT387 affects the ability of the two cell lines to form colonies over a period of 12 days. Figure 1c demonstrates that compared with those in the blank control and solvent groups, the cells treated with the drug at a concentration of $6 \mu \mathrm{M}$ had a markedly decreased number of colonies, and the colonies in the groups treated with the drug were much smaller than those in the blank control and DMSO groups $(\mathrm{P}<0.001)$. These data showed that CYT387 could significantly inhibit glioblastoma cell proliferation in vitro. 
a

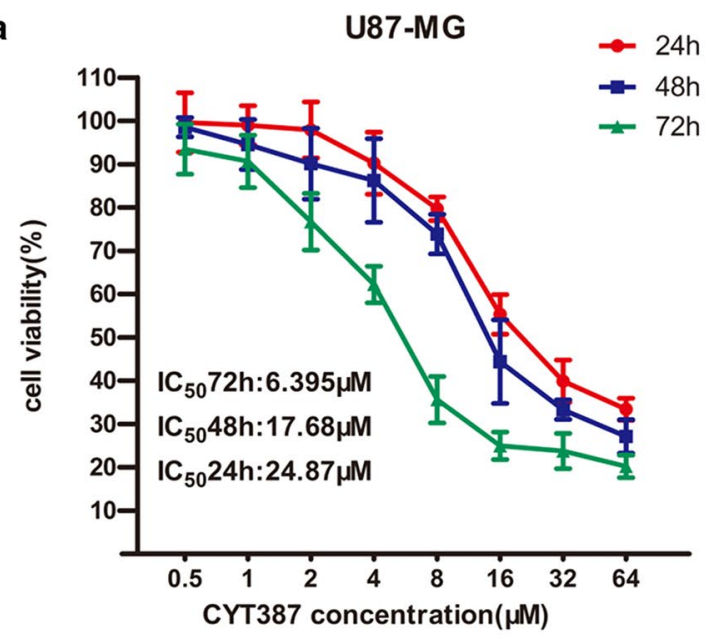

b

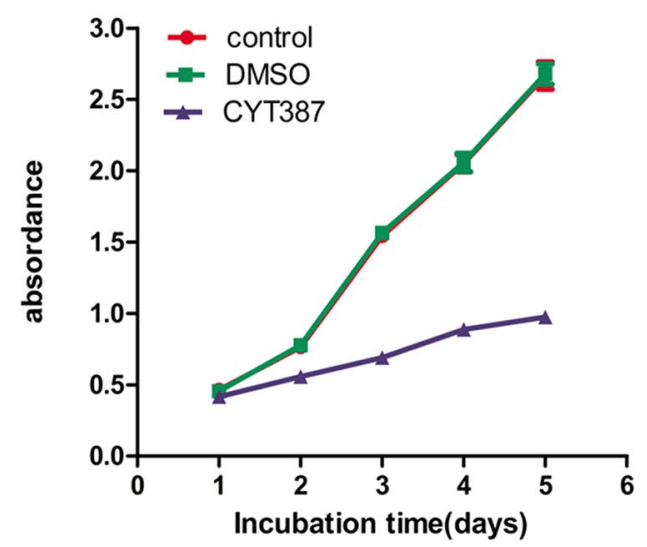

LN-229

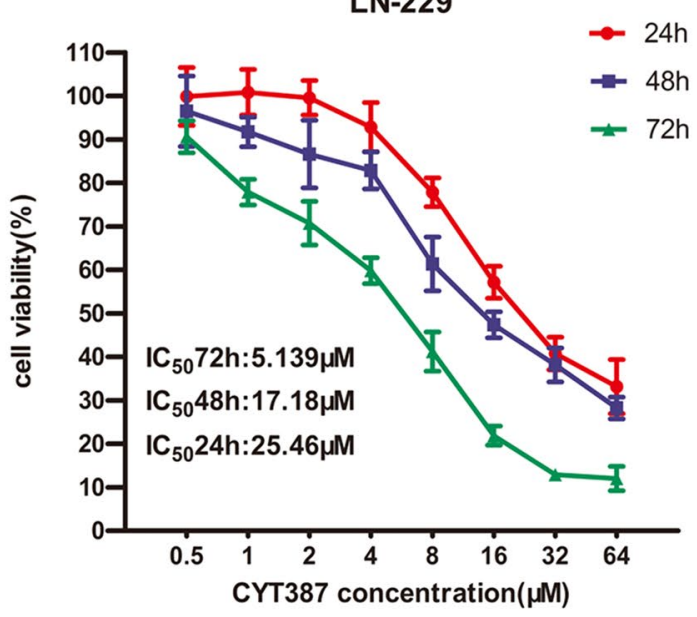

LN-229

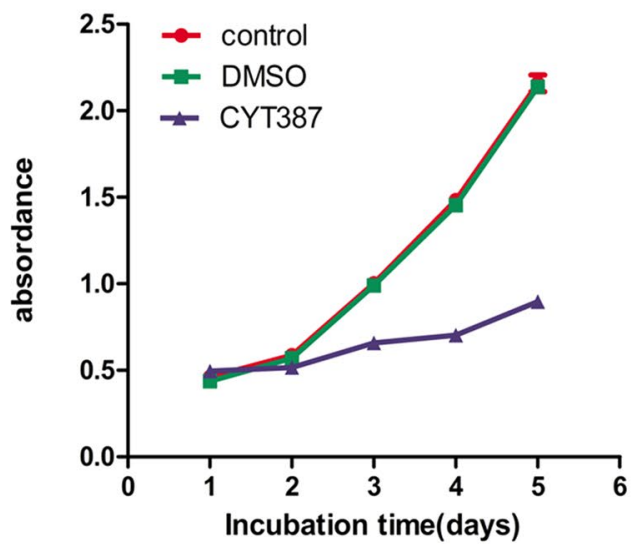

C

control DMSO

CYT387
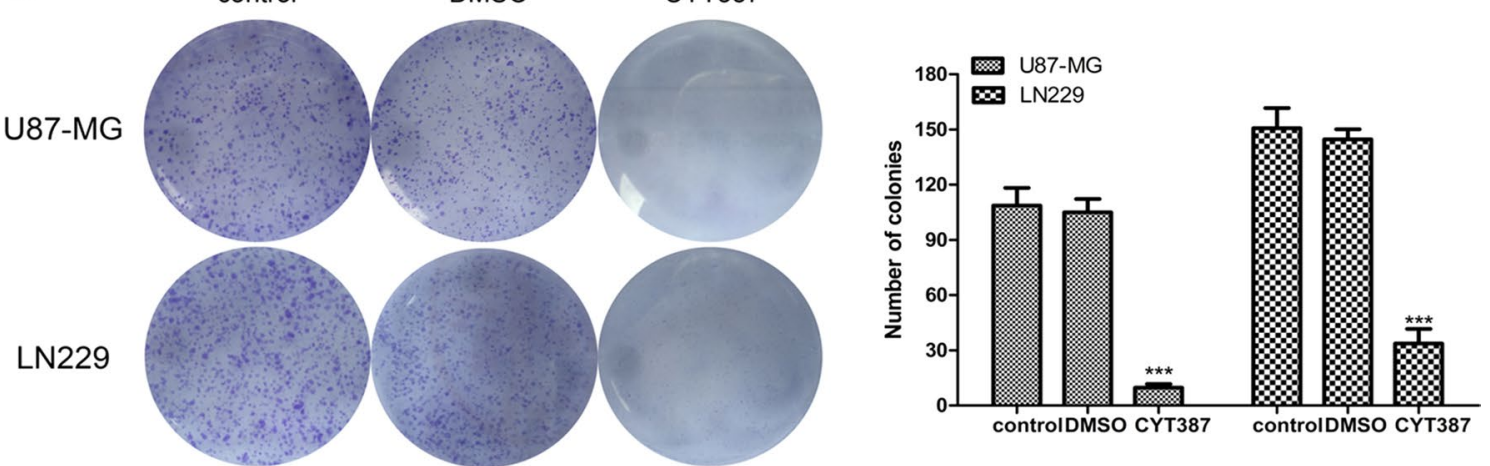

Fig. 1 CYT387 inhibits proliferation in glioblastoma cells. A Half maximal inhibitory concentration (IC50) of CYT387 was measured by CCK-8 in U87-MG and LN229 cells. B Glioblastoma cells treated with CYT387 dramatically decreased the capacity of proliferation using CCK-8 assay. C Colony formation assay showed decreased ability of forming colonies after treatment with CYT387. All experiments were repeated at least three times. $\left({ }^{*} p<0.05 ;{ }^{* *} p<0.01 ;{ }^{* * *} p<0.001\right)$

CYT387 dramatically inhibits the migration and invasion of glioblastoma cell lines in vitro

To explore whether CYT387 impacts tumor cell migration, a wound healing assay was adopted to assess the adherent tumor cell healing area at different times with drug treatment at a concentration of $5 \mu \mathrm{M}$. Compared with control or solvent treatment, CYT387 markedly inhibited the wound healing speed after only $24 \mathrm{~h}$, 
suggesting a poor migratory ability in the CYT387 group (Fig. 2a). Furthermore, we investigated the influence of CYT387 on the glioblastoma cell invasive capacity using a Transwell assay. The experimental results (Fig. 2b) showed that the average number of invaded cells in the group treated with a drug concentration of $6 \mu \mathrm{M}$ was significantly decreased compared to that in the blank control and solvent groups after $48 \mathrm{~h}$, showing that CYT387 had an inhibitory effect on the invasive ability of U87-MG and LN229 cells. To further research the detailed mechanism, two important matrix metalloproteinases (MMPs),
MMP2 and MMP9, were assessed by western blot analysis. As Fig. 2c shows, MMP2 and MMP9 expression levels were significantly reduced after tumor cells were treated with CYT387 for $48 \mathrm{~h}$ compared with control or DMSO treatment (Figure S2). These data fully demonstrated that CYT387 could effectively inhibit glioblastoma cell migration and invasion.

\section{CYT387 accelerates glioblastoma cell apoptosis}

Since apoptosis is critical to tumor regression, we performed Annexin V-FITC/PI staining using flow a

control
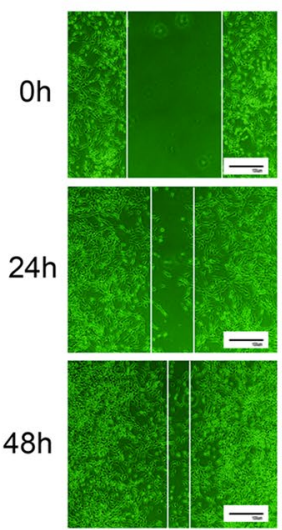

U87-MG

DMSO
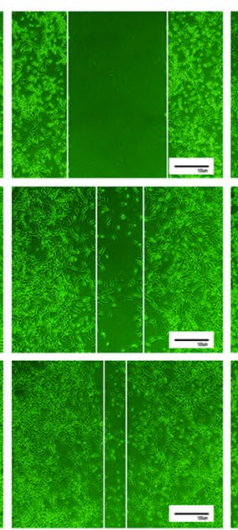

CYT387
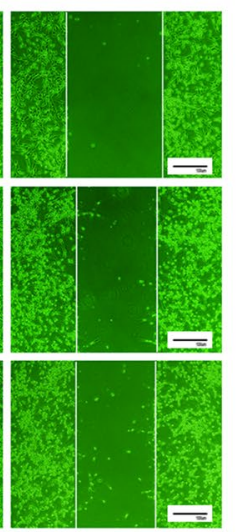

LN-229

DMSO
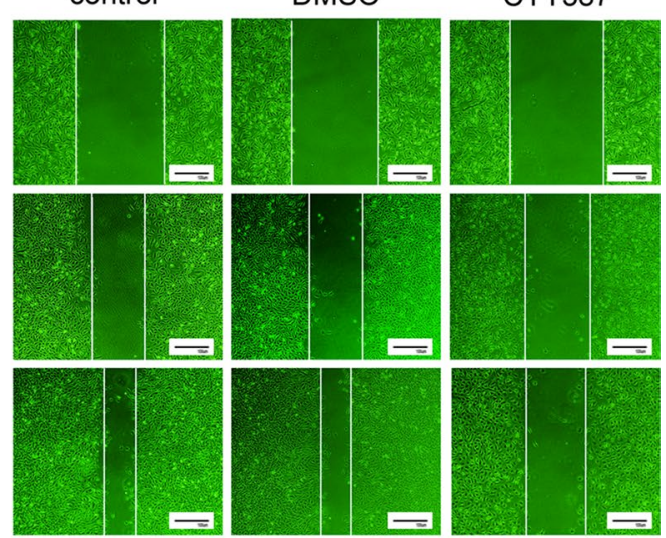

b

control

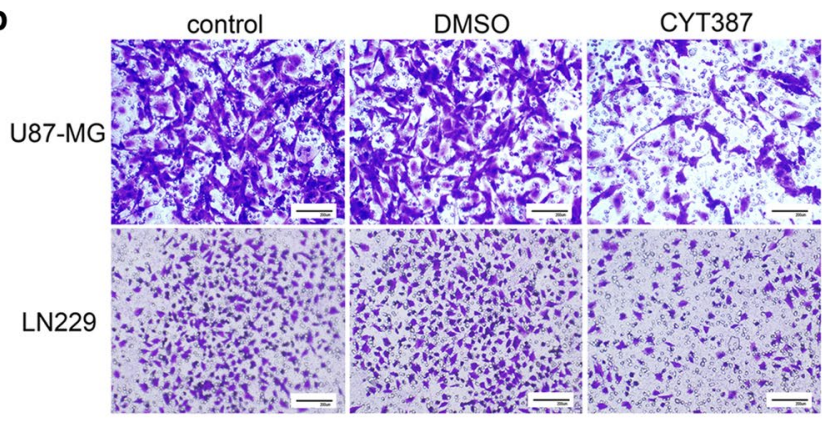

CYT387

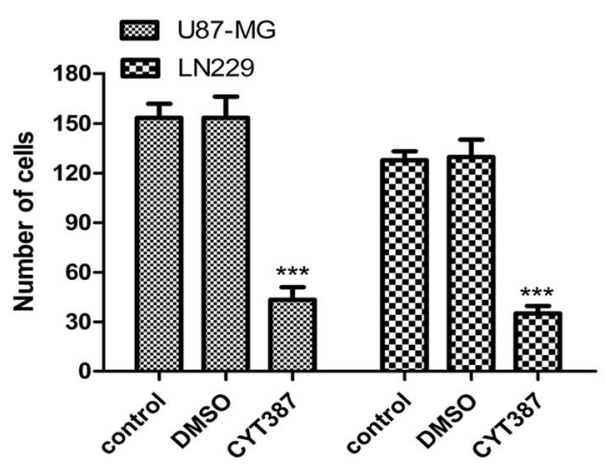

C

U87-MG

LN229

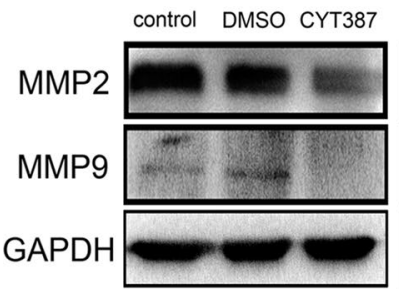

control DMSO CYT387

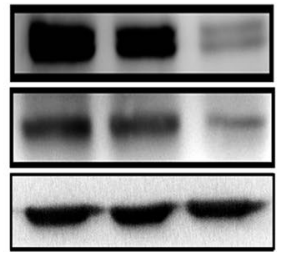

$72 \mathrm{kDa}$

$84 \mathrm{kDa}$

\section{$37 \mathrm{kDa}$}

Fig. 2 CYT387 inhibits migration and invasion in glioblastoma cells. A Migration ability of U87-MG and LN229 was measured by wound healing test after treated with CYT387. B Invasion capacity of glioblastoma cells was measured by transwell assay after treated with CYT387. C MMP2 and MMP9 were detected by western blot after treatment with CYT387. All experiments were repeated at least three times. $\left.{ }^{*} p<0.05 ;{ }^{* *} p<0.01 ;{ }^{* *} p<0.001\right)$ 
cytometry to test whether CYT387 can influence glioblastoma cell apoptosis. After cells were incubated with the drug for $24 \mathrm{~h}$ at a concentration of $5 \mu \mathrm{M}$, the tumor cell apoptosis rate was obviously increased compared with that observed in the blank control and DMSO groups (Fig. 3a, b). The apoptosis rates of U87-MG cells were $4.20 \pm 0.127 \%$ in the blank group, $4.47 \pm 0.287 \%$ in the DMSO group and $10.8 \pm 1.20 \%$ in the drug group $(\mathrm{P}<0.01)$, while the apoptosis rates of LN229 cells were $7.04 \pm 0.176 \%$ in the blank group, $7.21 \pm 0.138 \%$ in the DMSO group and $14.67 \pm 0.960 \%$ in the drug group $(\mathrm{P}<0.001)$. Moreover, the mechanism underlying the induction of apoptosis by CYT387 was assessed by western blot analysis. The protein expression of caspase- 9 was decreased, but that of cleaved caspase- 9 was increased in the groups treated with $5 \mu \mathrm{M}$ drug for $24 \mathrm{~h}$ compared with the blank control and DMSO groups (Fig. 3c and Figure S2). Additionally, the antiapoptotic protein $\mathrm{Bcl}-2$ and proapoptotic protein $\mathrm{Bax}$ were assessed. As shown in Fig. 3c, Bcl-2 expression was decreased, but Bax expression was increased after incubation with the drug $(6 \mu \mathrm{M})$ for $24 \mathrm{~h}$. All data indicated that CYT387 could obviously accelerate glioblastoma cell apoptosis.

\section{CYT387 arrests the glioblastoma cell cycle at the G2/M checkpoint}

We found that treatment with CYT387 $(5 \mu \mathrm{M})$ for 3 days induced cell cycle arrest at the G2/M checkpoint. As shown in Fig. 4a, b, the proportions of cells at the G2/M checkpoint for U87-MG cells were $23.23 \pm 0.54 \%$ in the drug group, $13.90 \pm 0.50 \%$ in the blank control group and $14.47 \pm 1.00 \%$ in the solvent group $(\mathrm{P}<0.001)$, while those for LN229 cells were $37.03 \pm 1.96 \%$ in the drug group, $15.11 \pm 0.39 \%$ in the blank control group and $15.44 \pm 0.79 \%$ in the solvent group $(\mathrm{P}<0.001)$. In

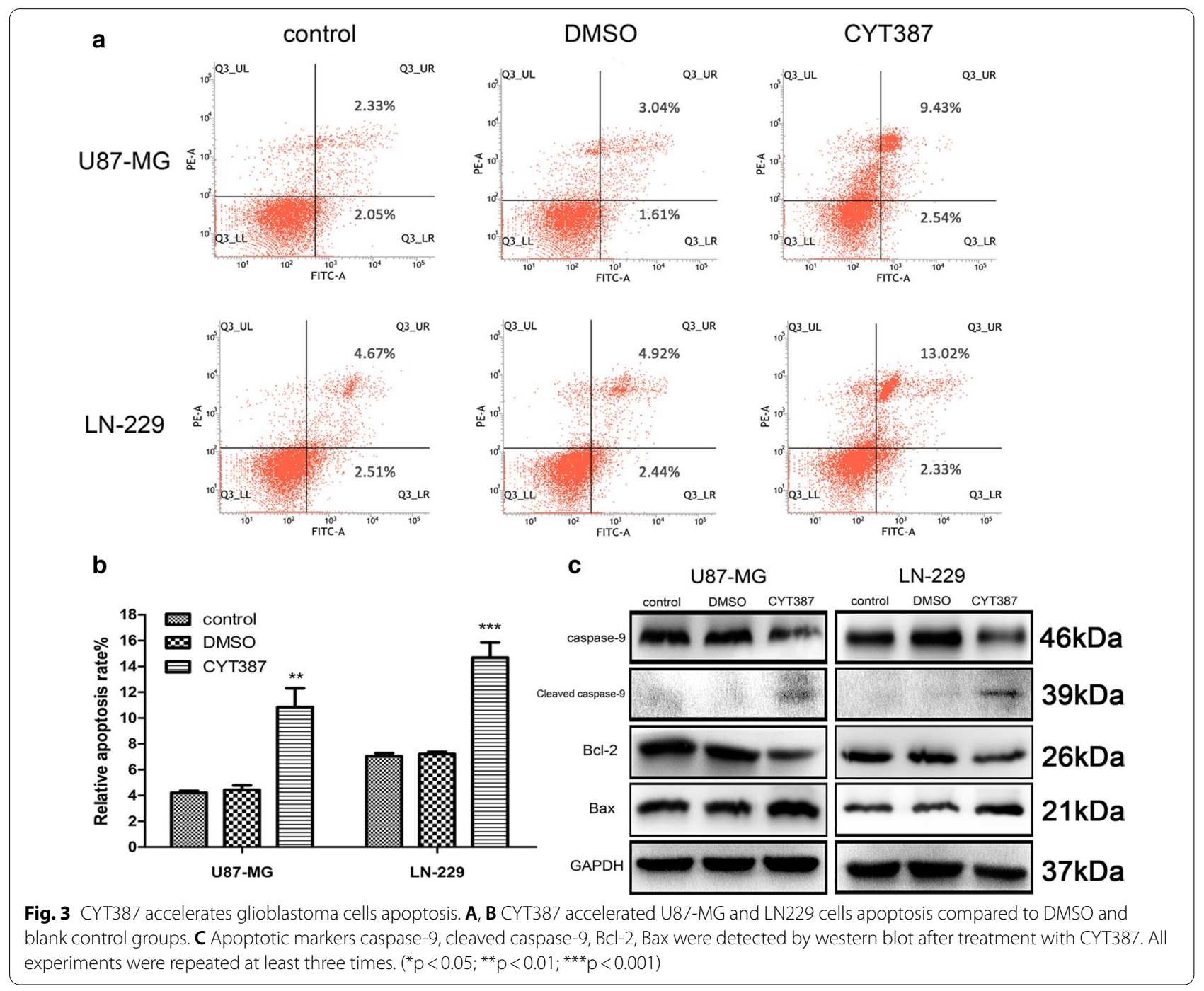




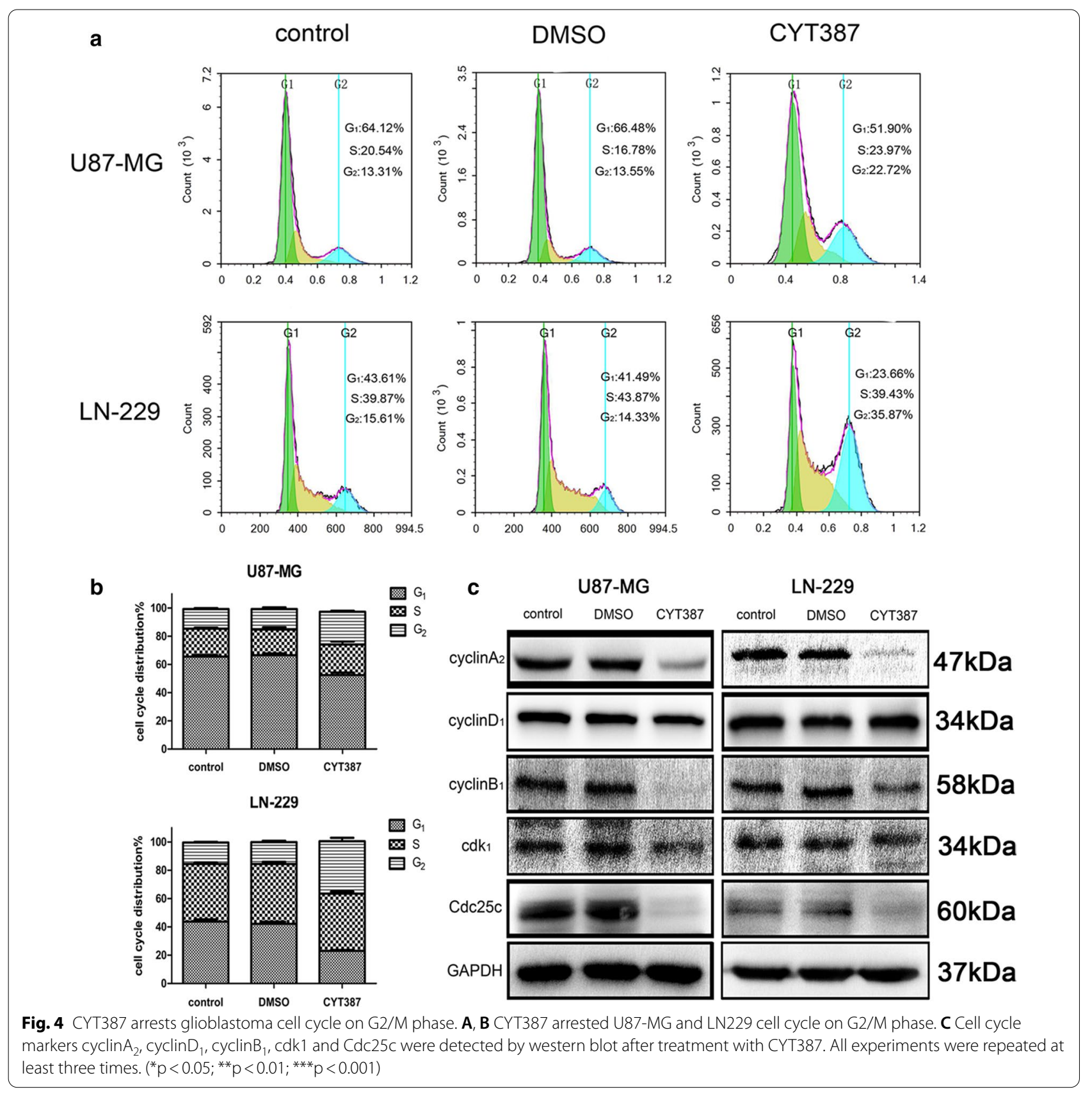

addition, the proportions of glioblastoma cells in the G1 and $\mathrm{S}$ phases were correspondingly decreased. In addition, we examined the expression levels of several cell cycle kinases including CyclinA2, CyclinD1, CyclinB1, Cdk1 and Cdc25c by western blot analysis. As shown in Fig. 4c, the expression of CyclinD1, an important regulator driving cell transition from the G1 phase into the $\mathrm{S}$ phase, exhibited a negligible change, while the levels of CyclinA2, CyclinB1, Cdk1 and Cdc25c, as several essential factors regulating the mitotic entry of cells from the
G2/M checkpoint, were significantly decreased in the drug group $(6 \mu \mathrm{M})$ compared to the blank control and DMSO groups (Figure S2). All this information suggested that CYT387 induced glioblastoma cell cycle arrest at the G2/M checkpoint to inhibit cell proliferation. 
CYT387 activates the Hippo signaling pathway by inhibiting IKBKE expression to suppress glioblastoma cell growth

The Hippo pathway, which is composed of a cascade of phosphorylated kinases, can regulate cell proliferation, apoptosis and differentiation. The core Hippo pathway has been well established in mammals. MST1/2 directly phosphorylates LATS1/2 with the help of Sav1 and Mob1. Then, LATS1/2 directly interacts with and phosphorylates YAP1 at Ser127, resulting in YAP sequestration in the cytoplasm and degradation via ubiquitylation by $14-3-3$ [24, 25]. When YAP1 is unphosphorylated, due to an ineffective Hippo pathway, it is transported into the nucleus to interact with TEAD1-4, creating transcription factors that induce the transcription of certain genes [29, 30], such as Axl [31], c-myc [32], Cyr61 [33, 34], and CTGF [34]. We showed that the levels of the core Hippo pathway effectors YAP1 and TEAD2 and their downstream factors including Axl or c-myc were decreased, while those of LATS2 and p-YAP1 (S127) were increased in the IKBKE-knockdown group compared with the blank control and scrambled groups by western blot analysis (Fig. 5a and Figure S3), indicating that IKBKE inhibition enhanced Hippo pathway activity by decreasing YAP1 and TEAD2 expression and increasing the expression of LATS2, which could accelerate the phosphorylation and degradation of YAP1. Additionally, overexpression of IKBKE increased YAP1 and TEAD2 expression, as well as the expression of the downstream factors Axl and c-myc, while reducing LATS2 and p-YAP1 (S127) expression (Fig. 5b and Figure S3). Furthermore, the effect of CYT387 on the Hippo pathway was confirmed to be mediated in a dose- and time-dependent manner by western blot analysis. As shown in Fig. 5c, with drug administration at a concentration of $6 \mu \mathrm{M}$ for $0 \mathrm{~h}, 24 \mathrm{~h}, 48 \mathrm{~h}$ or $72 \mathrm{~h}$, the expression of IKBKE, YAP1, TEAD2, Axl and c-myc gradually decreased over time; however, that of LATS2 and p-YAP1

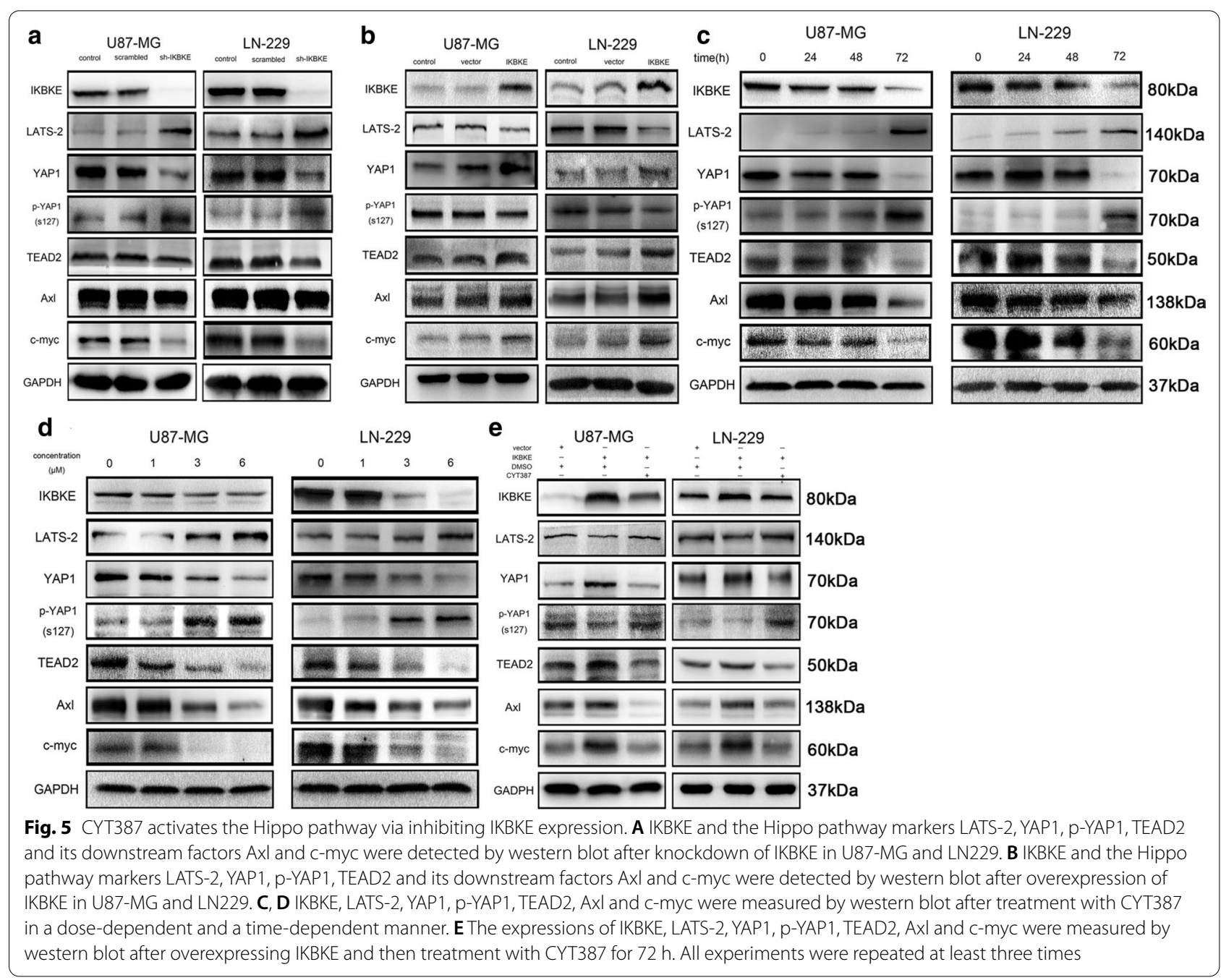


(S127) progressively increased in U87-MG and LN229 cells, with an increase particularly noted at $72 \mathrm{~h}$ (Figure S3). Moreover, as shown in Fig. 5d, when increasing concentrations including $0 \mu \mathrm{M}, 1 \mu \mathrm{M}, 3 \mu \mathrm{M}$ and $6 \mu \mathrm{M}$ were administered for $72 \mathrm{~h}$, the protein levels of IKBKE, YAP1, TEAD2, Axl and c-myc gradually decreased, while those of LATS2 and p-YAP1 (S127) increased, especially for the drug concentrations over $3 \mu \mathrm{M}$ (Figure S3). However, it should be noted that the decrease in IKBKE expression resulting from CYT387 treatment was mainly due to posttranslational protein modification rather than effects at the transcriptional level, as IKBKE mRNA levels were determined to be negligibly changed by using real-time RT-PCR to evaluate dose- and time-dependent effects on U87-MG and LN229 cells (Additional file 1: Figure S1a, b). We next verified that CYT387 could reverse the inhibition of the Hippo pathway resulting from the overexpression of IKBKE. As shown in Fig. 5e, the expression levels of IKBKE, YAP1, TEAD2, Axl, and c-myc were increased with IKBKE overexpression and then decreased with $6 \mu \mathrm{M}$ CYT387 treatment for $72 \mathrm{~h}$. However, the expression trends for LATS-2 and p-YAP1 (S127) were contrary to those for YAP1 and TEAD2 (Figure S3). All of the above data demonstrated that CYT387 could enhance the activity of the Hippo pathway, which reduced YAP1, TEAD2 and downstream target protein expression, to inhibit glioblastoma progression by suppressing and inactivating IKBKE.

\section{IKBKE may directly interact with TEAD2 and YAP1 to regulate the Hippo pathway, accelerating TEAD2 and YAP1 translocation into the nucleus}

To investigate the detailed mechanism underlying the impact of IKBKE on the Hippo pathway, we first confirmed that IKBKE regulates YAP1 and TEAD2 at the posttranslational level. As shown in Fig. 6a, the mRNA expression of TEAD2 and YAP1 was hardly changed in the IKBKE-knockdown group compared to the blank control and scrambled groups, showing that inhibition of IKBKE decreased YAP1 and TEAD2 levels via posttranslational modification rather than changes in the mRNA levels. Next, we verified that IKBKE can directly interact with TEAD2 and YAP1 using endogenous co-IP (Fig. 6b and Figure S4). Then, we used Flag-IKBKE and HA-TEAD2 plasmids to perform exogenous co-IP. As shown in Fig. 6c, Flag-IKBKE could directly interact with HA-TEAD2 (Figure S4). We also performed exogenous co-IP using Flag-IKBKE and HA-YAP1 plasmids, showing that Flag-IKBKE interacted with HA-YAP1 (Fig. 6d and Figure S4). Additionally, we demonstrated that inhibition of IKBKE suppressed TEAD2 and YAP1 translocation into the nucleus using western blot analysis (Fig. 6e and Figure S4). All these data showed that IKBKE

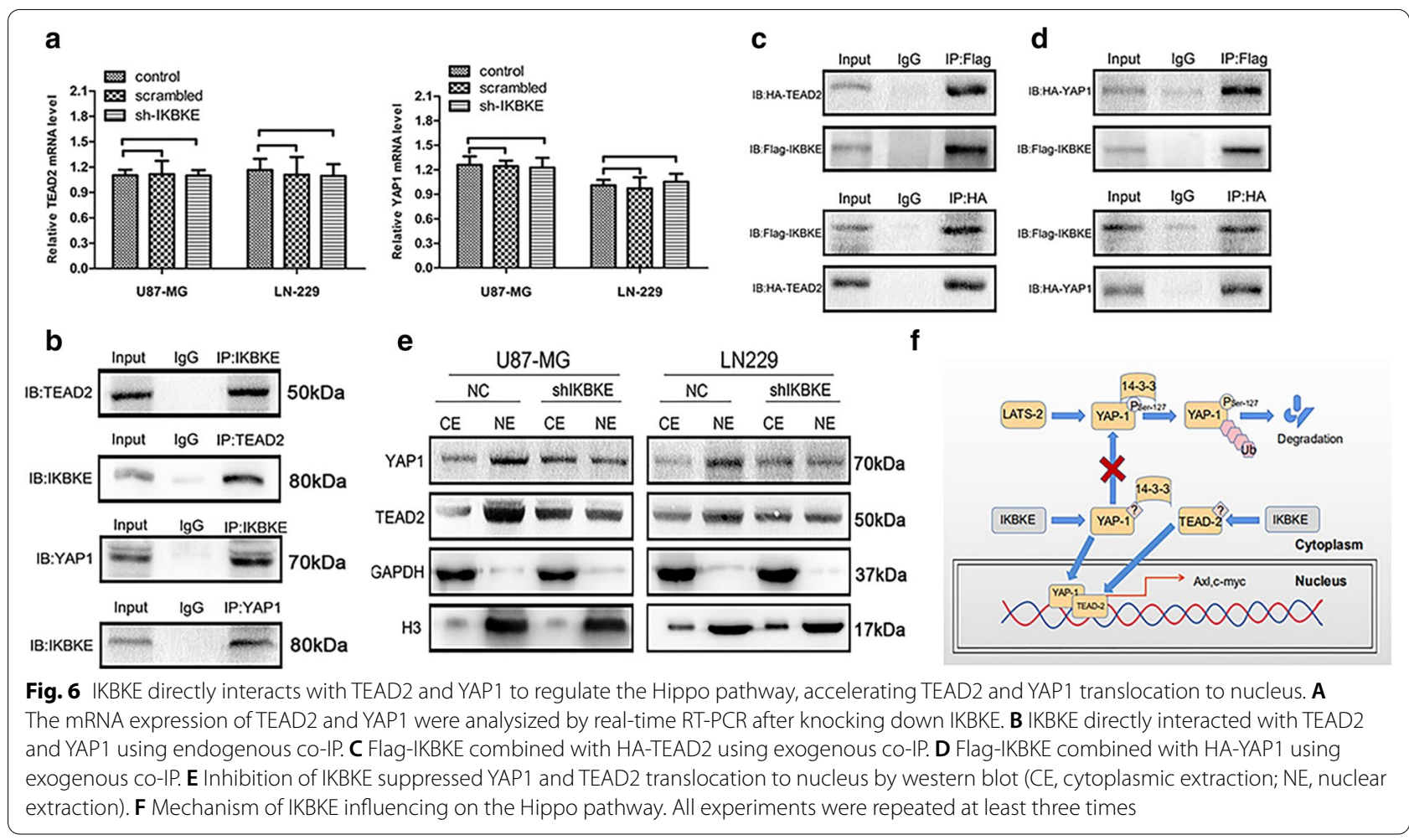


may directly interact with YAP1 and TEAD2 to promote YAP1 and TEAD2 transport into the nucleus. The detailed pathway diagram is shown in Fig. $6 f$.
CYT387 inhibits tumor growth in a subcutaneous nude mouse model but has little impact on an intracranial orthotopic model

To confirm whether CYT387 can inhibit tumor growth in vivo, we first established a subcutaneous nude mouse model via U87-MG cell inoculation. We used 6 mice fed

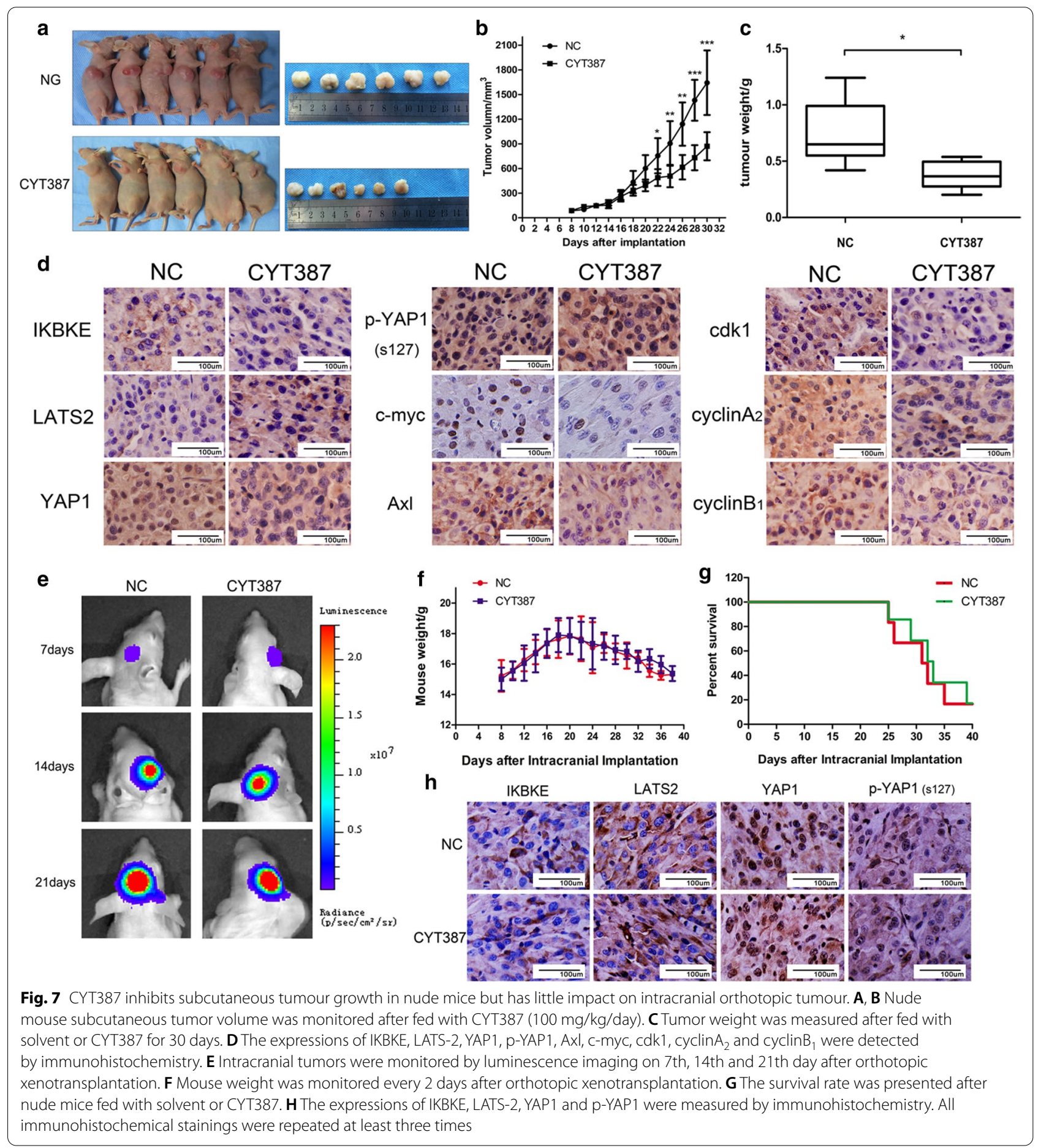


the same concentration of solvent as the negative control (NC) group and 6 mice fed CYT387 at a dose of $100 \mathrm{mg} /$ $\mathrm{kg} /$ day as the experimental group. Tumor volume was monitored every two days, and all mice were euthanized to measure implanted tumor weight on the 30th day. As time passed, the size of the tumors in the mice treated with CYT387 was obviously decreased (Fig. 7a, b) $(\mathrm{P}<0.05)$, and the weight of these tumors was lower than that of the tumors in the NC group (Fig. $7 \mathrm{c})(\mathrm{P}<0.05)$. Then, the expression of IKBKE, YAP1, Axl and c-myc was found to be downregulated, while that of LATS2 and p-YAP1 (S127) was shown to be increased in the tumors in the CYT387 $(100 \mathrm{mg} / \mathrm{kg} /$ day $)$ group compared with those in the NC group by immunohistochemical staining (Fig. 7d). Additionally, the levels of cell cycle markers such as Cdk1, CyclinA2 and CyclinB1 were reduced after mice were fed CYT387 compared with $\mathrm{NC}$ treatment (Fig. 7d). To explore whether CYT387 has an inhibitory effect on intracranial glioblastoma-like subcutaneously implanted tumors, we next established an orthotopic xenograft model with nude mice divided into two groups: an NC group fed the same concentration of solvent and a drug group fed CYT387 (100 mg/kg/day); U87-MG cells infected with a luciferase-expressing lentivirus were used. Imaging of intracranial tumor size was performed every 7 days after orthotopic xenotransplantation, and mouse weight was measured every 2 days. As shown in Fig. 7e, luminescence imaging showed no obvious differences between the NC group and the drug group at 7, 14, and 21 days. The weights and survival rates of the two groups of mice also showed few significant differences (Fig. 7f, g). Furthermore, we assessed IKBKE, LATS2, YAP1, and p-YAP1 (S127) expression levels by immunohistochemical staining, showing that there were few significant differences in these targets between the drug group and the $\mathrm{NC}$ group (Fig. 7h). These data suggested that CYT387 could inhibit glioblastoma progression in subcutaneous tumors but had little effect on intracranial orthotopic xenografts.

\section{Discussion}

CYT387 (momelotinib), a JAK1/2 kinase inhibitor tested in clinical trials for myelofibrosis, has recently been identified as a potent IKBKE inhibitor. Zhu et al. [22] revealed that CYT387, which disrupts a cytokine circuit involving CCL5, IL-6, and STAT3, could suppress KRAS-dependent lung cancer cell growth. Barbie et al. [23] reported that CYT387 inhibited breast cancer proliferation via IKBKE, inducing NF-KB and STAT3 activation, while inhibition of JAK alone did not have the same inhibitory effect. JAK/STAT3 signaling has also been demonstrated to be central to GBM, and many smallmolecule JAK inhibitors have produced positive results in in vitro and in vivo studies of GBM. In view of these findings, CYT387 may be a promising candidate inhibitor for gliomas due to its dual targeting. Here, we found that CYT387 decreased IKBKE expression levels in a doseand time-dependent manner, especially with drug treatment for $72 \mathrm{~h}$. However, this action may be dominated by posttranslational protein modification rather than changes at the transcriptional level, given that real-time RT-PCR analysis showed that IKBKE mRNA expression was negligibly altered by treatment with CYT387 (Additional file 1: Figure S1a, b). We also confirmed that CYT387 suppressed glioblastoma cell proliferation, migration, and invasion; promoted cell apoptosis; and induced cell cycle arrest at the G2/M checkpoint in vitro.

In this paper, we focused on exploring the novel mechanism by which CYT387, as a potent IKBKE inhibitor, inhibits the malignant progression of human glioblastoma. Recent studies have shown that IKBKE dominates cancer progression induced by the NF- $\mathrm{kB}$ pathway. For example, IKBKE phosphorylates CYLD and TRAF2 in breast cancer cells, which induces NF- $\mathrm{kB}$ activation and contributes to cell transformation [35, 36]. Guo et al. [37] also showed that IKBKE repressed FOXO3a primarily through direct phosphorylation of Ser644, which was found to promote cell survival, growth and tumorigenesis. In this paper, we proposed that IKBKE can regulate the Hippo pathway by directly interacting with YAP1 and TEAD2 and promoting YAP1 and TEAD2 transport into the nucleus. Previous data indicated that the Hippo signaling pathway might contribute to glioblastoma progression. Ji et al. [26] reported that LATS1 expression is significantly downregulated in glioma; furthermore, reduced LATS1 expression is markedly negatively correlated with the WHO grade and overall survival time. Orr et al. [27] demonstrated that elevated nuclear immunoreactivity of YAP1 was prominent in high-grade gliomas, suggesting the potential role of YAP1 in the pathobiology of the most common malignant brain tumors. Therefore, we speculated that IKBKE may promote glioblastoma progression via regulation of the Hippo pathway.

Our studies also showed that CYT387 decreased YAP1 and TEAD2 expression and increased LATS-2 and p-YAP1 (S127) expression to strengthen Hippo pathway activity mainly induced by inactivation of IKBKE. We also found that IKBKE knockdown enhanced Hippo pathway activity predominantly through direct interactions with YAP1 and TEAD2, which inhibited YAP1 and TEAD2 transport into the nucleus. Recently, Lue et al. [20] showed that CYT387 inhibited total YAP1 expression and enhanced p-YAP1 (S127) expression in renal cell carcinoma cell lines, and we discovered, for the first time, that this function was mainly induced by inhibition of IKBKE. LATS1/2 expression can be dominated by 
YAP expression via a negative feedback loop in the Hippo pathway [38, 39].

Recent studies have reported that caspase-9 triggers cell apoptosis following cleavage and activation after being sensitized by apoptosomes [40, 41]. We also verified by western blot analysis that the antiapoptotic protein Bcl-2 and total caspase- 9 levels were decreased, while those of proapoptotic Bax and cleaved caspase-9 were increased by drug treatment, demonstrating that CYT387 could promote glioblastoma cell apoptosis. Furthermore, we showed that CYT387 induced cell cycle arrest at the G2/M checkpoint. According to previous studies, CyclinA2 participates in the regulation of the $\mathrm{S}$ phase as well as mitotic entry and is also a marker of cell proliferation and invasion [42]. The CyclinB1-Cdk1 complex, a key regulator of mitotic entry, dominates mitosis skipping, arresting cells at the G2/M checkpoint via inactivation of Cdk1 kinase by degradation of cyclinB1 [43]. Cdc25C can fully activate CyclinB1-Cdk1 after translocation into the nucleus by directly dephosphorylating Cdk1 to induce G2/M progression [44, 45]. Through western blot analysis, CyclinA2, CyclinB1, Cdk1 and $\mathrm{Cdc} 25 \mathrm{c}$ expression was found to be obviously decreased after drug treatment, while the expression of CyclinD1, an essential factor that regulates the cell cycle transition from the G1 to $S$ phase [46], was negligibly altered, suggesting that cells treated with CYT387 undergo cell cycle arrest at the G2/M checkpoint. In previous investigations, similar results were obtained, showing that increased LATS1 expression inhibited cell proliferation by blocking the G2/M transition, mainly through inhibition of the kinase activity of the $\mathrm{Cdc} 2 / \mathrm{Cyclin} \mathrm{A} / \mathrm{B}$ complex [47].

In a following experiment, we verified that CYT387 could inhibit tumor growth in a subcutaneous nude mouse model but found that CYT387 had little effect on an intracranial orthotopic model. The expression of IKBKE, YAP1 and Hippo downstream factors such as c-myc and Axl was found to be decreased, while the expression of LATS-2 and p-YAP (S127) was shown to be increased in the drug group using immunohistochemical staining, indicating Hippo pathway activity in subcutaneous tumors was enhanced after drug treatment. However, no obvious changes in IKBKE or Hippo activity were observed in the intracranial xenografts. We hypothesized that this was probably due to the limited ability of CYT387 to penetrate the blood-brain barrier (BBB). According to previous research, Durmus et al. [48] reported that the cerebral concentration of CYT387 was far lower than that in the plasma but that it was dramatically increased in Bcrp1-/-; Mdr1a/1b-/- mice compared with WT mice, while the plasma concentration was little impacted, demonstrating that cerebral accumulation of CYT387 is likely restricted by Mdr1a/1b and Bcrp1. Therefore, it is possible that CYT387 does not reach an effective concentration in the brain to suppress intracranial glioblastoma progression, and the ability of this drug to pass through the blood-brain barrier (BBB) urgently needs to be improved. A recent study showed that lipid-core nanocapsules act as drug shuttles through the $\mathrm{BBB}$, delivering drugs to the brain tissue with high efficiency and reducing glioblastoma after intravenous or oral administration [49]. Thus, in the future, CYT387 will be encapsulated in nanocapsules for shuttling across the $\mathrm{BBB}$ to enable it to exert an antitumor effect.

\section{Supplementary Information}

The online version contains supplementary material available at https://doi. org/10.1186/s12967-021-03070-3.

Additional file 1: Figure S1. CYT387 hardly changes IKBKE mRNA expression in a dose-dependent and a time-dependent manner. Figure S2. Original western blots used in the Figs. 2, 3 and 4. Figure S3. Original western blots used in the Fig. 5. Figure S4. Original western blots used in the Fig. 6.

\section{Acknowledgements}

Not applicable.

\section{Authors' contributions}

$J \mathrm{~L}$ and XW completed the mechanism experiment. $J$ finished the cell phenotype experiments. GCG and YL finished the in vivo experiments. JL completed statistical work. XW and $\mathrm{QH}$ were responsible for experimental design and technical support. All authors read and approved the final manuscript.

\section{Funding}

This work is supported by the National Nature Science Foundation of China, Grant No. 81572490

And the National Nature Science Foundation of China, Grant No.81172405.

\section{Availability of data and materials}

The data that support the findings of this study are available from the corresponding author upon reasonable request. And all WB bands have been added to Additional file 1: Figure S1.

\section{Declarations}

\section{Ethics approval and consent to participate}

The Animal Ethics and Welfare Committee of Shandong Cancer Hospital and Institute had provided approval before all the animal experiments performed and all the participants had written informed consent before enrollment.

\section{Consent for publication}

Not applicable.

Competing interests

The authors declare that they have no competing interests.

\section{Author details}

${ }^{1}$ Department of Radiation Oncology, Shandong Cancer Hospital and Institute, Shandong First Medical University and Shandong Academy of Medical Sciences, Jinan, Shandong, China. ${ }^{2}$ Department of Neurosurgery, The First Affiliated Hospital of Shandong First Medical University \& Shandong Provincial Qianfoshan Hospital, Shandong Medicine and Health Key Laboratory 
of Neurosurgery, Jinan, Shandong, China. ${ }^{3}$ Department of Nursing, The First Affiliated Hospital of Shandong First Medical University \& Shandong Provincial Qianfoshan Hospital, Jinan, Shandong, China. ${ }^{4}$ Department of Neurosurgery, Renmin Hospital of Henan Province, Zhengzhou, Henan, China. ${ }^{5}$ Department of Neurosurgery, Tianjin Medical University General Hospital, Tianjin, China. ${ }^{6}$ Tianjin Neurological Institute, Key Laboratory of Post-Neuroinjury Neuro-repair and Regeneration in Central Nervous System, Ministry of Education and Tianjin City, Tianjin, People's Republic of China.

Received: 30 May 2021 Accepted: 2 September 2021

Published online: 20 September 2021

\section{References}

1. Stupp R, Mason WP, van den Bent MJ, Weller M, Fisher B, Taphoorn MJ, et al. Radiotherapy plus concomitant and adjuvant temozolomide for glioblastoma. N Engl J Med. 2005;352:987-96.

2. Stupp R, Hegi ME, Mason WP, van den Bent MJ, Taphoorn MJ, Janzer RC, et al. Effects of radiotherapy with concomitant and adjuvant temozolomide versus radiotherapy alone on survival in glioblastoma in a randomised phase III study: 5-year analysis of the EORTC-NCIC trial. Lancet Oncol. 2009;10:459-66.

3. Wang X, Lu J, Guo G, Yu J. Immunotherapy for recurrent glioblastoma: practical insights and challenging prospects. Cell Death Dis. 2021;12(4):299.

4. Wang X, Guo G, Guan H, Yu Y, Lu J, et al. Challenges and potential of PD-1/ PD-L1 checkpoint blockade immunotherapy for glioblastoma. J Exp Clin Cancer Res. 2019;38(1):87.

5. Häcker H, Karin M. Regulation and function of IKK and IKK-related kinases. Sci STKE. 2006;357:re13.

6. Shih VF, Tsui R, Caldwell A, Hoffmann A. A single NF-kB system for both canonical and non-canonical signaling. Cell Res. 2011;21:86-102.

7. Boehm JS, Zhao JJ, Yao J, Kim SY, Firestein R, Dunn IF. Integrative genomic approaches identify IKBKE as a breast cancer oncogene. Cell. 2007;129:1065-79.

8. Guan H, Zhang H, Cai J, Wu J, Yuan J, Li J, et al. IKBKE is over-expressed in glioma and contributes to resistance of glioma cells to apoptosis via activating NF-KB. J Pathol. 2011;223:436-45.

9. Li H, Chen L, Zhang A, Wang G, Han L, Yu K, et al. Silencing of IKKepsilon using siRNA inhibits proliferation and invasion of glioma cells in vitro and in vivo. Int J Oncol. 2012;41:169-78.

10. Lu J, Yang Y, Guo G, Liu Y, Zhang Z, Dong S, et al. IKBKE regulates cell proliferation and epithelial-mesenchymal transition of human malignant glioma via the Hippo pathway. Oncotarget. 2017;8:49502-14.

11. Guo JP, Shu SK, He L, Lee YC, Kruk PA, Grenman S, et al. Deregulation of IKBKE is associated with tumour progression, poor prognosis, and cisplatin resistance in ovarian cancer. Am J Pathol. 2009;175:324-33.

12. Péant B, Diallo JS, Dufour F, Le Page C, Delvoye N, Saad F, et al. Overexpression of IKB-kinase- $\varepsilon$ (IKKE/IKKi) induces secretion of inflammatory cytokines in prostate cancer cell lines. Prostate. 2009;69:706-18.

13. Guo J, Kim D, Gao J, Kurtyka C, Chen H, Yu C, et al. IKBKE is induced by STAT3 and tobacco carcinogen and determines chemosensitivity in nonsmall cell lung cancer. Oncogene. 2013;32:151-9.

14. Li W, Chen Y, Zhang J, Hong L, Yuan N, Wang X, et al. IKBKE upregulation is positively associated with squamous cell carcinoma of the lung in vivo and malignant transformation of human bronchial epithelial cells in vitro. Med Sci Monit. 2015;21:1577-86.

15. Lee SE, Hong M, Cho J, Lee J, Kim KM. IKKE and TBK1 expression in gastric cancer. Oncotarget. 2017;8:16233-42.

16. Hildebrandt MA, Tan W, Tamboli P, Huang M, Ye Y, Lin J, et al. Kinome expression profiling identifies IKBKE as a predictor of overall survival in clear cell renal cell carcinoma patients. Carcinogenesis. 2012;33:799-803.

17. Wang X, Teng F, Lu J, Mu D, Zhang J, Yu J. Expression and prognostic role of IKBKE and TBK1 in stage I non-small cell lung cancer. Cancer Manag Res. 2019;11:6593-602.

18. Yin M, Wang $X$, Lu J. Advances in IKBKE as a potential target for cancer therapy. Cancer Med. 2019;9:247-58.
19. Tyner JW, Bumm TG, Deininger J, Wood L, Aichberger KJ, Loriaux MM, et al. CYT387, a novel JAK2 inhibitor, induces hematologic responses and normalizes inflammatory cytokines in murine myeloproliferative neoplasms. Blood. 2010;115:5232-40.

20. Lue HW, Cole B, Rao SA, Podolak J, Van Gaest A, King C, et al. Src and STAT3 inhibitors synergize to promote tumour inhibition in renal cell carcinoma. Oncotarget. 2015;6:44675-87.

21. Hu Y, Dong XZ, Liu X, Liu P, Chen YB. Enhanced antitumour activity of cetuximab in combination with the Jak inhibitor CYT387 against non-small-cell lung cancer with various genotypes. Mol Pharm. 2016;13:689-97.

22. Zhu Z, Aref AR, Cohoon TJ, Barbie TU, Imamura Y, Yang S, et al. Inhibition of KRAS-driven tumourigenicity by interruption of an autocrine cytokine circuit. Cancer Discov. 2014;4:452-65.

23. Barbie TU, Alexe G, Aref AR, Li S, Zhu Z, Zhang X, et al. Targeting an IKBKE cytokine network impairs triple-negative breast cancer growth. J Clin Invest. 2014;124:5411-23.

24. Yu FX, Guan KL. The Hippo pathway: regulators and regulations. Genes Dev. 2013;27:355-71.

25. Yu FX, Zhao B, Guan KL. Hippo pathway in organ size control, tissue homeostasis, and cancer. Cell. 2015;163:811-28.

26. Ji T, Liu D, Shao W, Yang W, Wu H, Bian X. Decreased expression of LATS1 is correlated with the progression and prognosis of glioma. J Exp Clin Cancer Res. 2012;31:67.

27. Orr BA, Bai H, Odia Y, Jain D, Anders RA, Eberhart CG. Yes-associated protein 1 is widely expressed in human brain tumours and promotes glioblastoma growth. J Neuropathol Exp Neurol. 2011;70:568-77.

28. Liu Y, Lu J, Zhang Z, Zhu L, Dong S, Guo G, et al. Amlexanox, a selective inhibitor of IKBKE, generates anti-tumoral effects by disrupting the Hippo pathway in human glioblastoma cell lines. Cell Death Dis. 2017;8:e3022.

29. Harvey KF, Zhang X, Thomas DM. The Hippo pathway and human cancer. Nat Rev Cancer. 2013;13:246-57.

30. Pobbati AV, Hong W. Emerging roles of TEAD transcription factors and its coactivators in cancers. Cancer Biol Ther. 2013;14:390-8.

31. Xu MZ, Chan SW, Liu AM, Wong KF, Fan ST, Chen J, et al. AXL receptor kinase is a mediator of YAP-dependent oncogenic functions in hepatocellular carcinoma. Oncogene. 2011;30:1229-40.

32. Neto-Silva RM, de Beco S, Johnston LA. Evidence for a growth-stabilizing regulatory feedback mechanism between Myc and Yorkie, the Drosophila homolog of YAP. Dev Cell. 2010;19:507-20.

33. Lai $D$, Ho KC, Hao Y, Yang $X$. Taxol resistance in breast cancer cells is mediated by the hippo pathway component TAZ and its downstream transcriptional targets Cyr61 and CTGF. Cancer Res. 2011;71:2728-38.

34. Zhang H, Liu CY, Zha ZY, Zhao B, Yao J, Zhao S, et al. TEAD transcription factors mediate the function of TAZ in cell growth and epithelial-mesenchymal transition. J Biol Chem. 2009;284:13355-62.

35. Shen RR, Zhou AY, Kim E, Lim E, Habelhah H, Hahn WC. IkB kinase $\varepsilon$ phosphorylates TRAF2 to promote mammary epithelial cell transformation. Mol Cell Biol. 2012;32:4756-68.

36. Hutti JE, Shen RR, Abbott DW, Zhou AY, Sprott KM, Asara JM, et al. Phosphorylation of the tumor suppressor CYLD by the breast cancer oncogene IKKepsilon promotes cell transformation. Mol Cell. 2009;34:461-72.

37. Guo JP, Tian W, Shu S, Xin Y, Shou C, Cheng JQ. IKBKE phosphorylation and inhibition of FOXO3a: a mechanism of IKBKE oncogenic function. PLOS ONE. 2013;8:e63636.

38. Moroishi T, Park HW, Qin B, Chen Q, Meng Z, Plouffe SW, et al. A YAP/TAZinduced feedback mechanism regulates Hippo pathway homeostasis. Genes Dev. 2015;29:1271-84.

39. Dai X, Liu H, Shen S, Guo X, Yan H, Ji X, et al. YAP activates the Hippo pathway in a negative feedback loop. Cell Res. 2017;27:1073.

40. Kim B, Srivastava SK, Kim SH. Caspase-9 as a therapeutic target for treating cancer. Expert Opin Ther Targets. 2015;19:113-27.

41. Shalini S, Dorstyn L, Dawar S, Kumar S. Old, new and emerging functions of caspases. Cell Death Differ. 2015:22:526-39.

42. Loukil A, Cheung CT, Bendris N, Lemmers B, Peter M, Blanchard JM. Cyclin A2: at the crossroads of cell cycle and cell invasion. World J Biol Chem. 2015;6:346-50. 
43. Nakayama Y, Yamaguchi N. Role of cyclin B1 levels in DNA damage and DNA damage-induced senescence. Int Rev Cell Mol Biol. 2013;305:303-37.

44. Sur S, Agrawal DK. Phosphatases and kinases regulating CDC25 activity in the cell cycle: clinical implications of CDC25 overexpression and potential treatment strategies. Mol Cell Biochem. 2016;416:33-46.

45. Aressy B, Ducommun B. Cell cycle control by the CDC25 phosphatases. Anticancer Agents Med Chem. 2008;8:818-24.

46. Qie S, Diehl JA. Cyclin D1, cancer progression, and opportunities in cancer treatment. J Mol Med (Berl). 2016;94:1313-26.

47. Visser S, Yang X. LATS tumor suppressor: a new governor of cellular homeostasis. Cell Cycle. 2010;9:3892-903.

48. Durmus S, Xu N, Sparidans RW, Wagenaar E, Beijnen JH, Schinkel AH. P-glycoprotein (MDR1/ABCB1) and breast cancer resistance protein
(BCRP/ABCG2/M) restrict brain accumulation of the JAK1/2 inhibitor, CYT387. Pharmacol Res. 2013;76:9-16.

49. Rodrigues SF, Fiel LA, Shimada AL, Pereira NR, Guterres SS, Pohlmann AR, et al. Lipid-core nanocapsules act as a drug shuttle through the blood brain barrier and reduce glioblastoma after intravenous or oral administration. J Biomed Nanotechnol. 2016;12:986-1000.

\section{Publisher's Note}

Springer Nature remains neutral with regard to jurisdictional claims in published maps and institutional affiliations.
Ready to submit your research? Choose BMC and benefit from:

- fast, convenient online submission

- thorough peer review by experienced researchers in your field

- rapid publication on acceptance

- support for research data, including large and complex data types

- gold Open Access which fosters wider collaboration and increased citations

- maximum visibility for your research: over $100 \mathrm{M}$ website views per year

At BMC, research is always in progress.

Learn more biomedcentral.com/submissions 